\title{
BMJ Open Prayer as a pain intervention: protocol of a systematic review of randomised controlled trials
}

\author{
Alexandra Ferreira-Valente (10) ,1,2 Margarida Jarego, ${ }^{1}$ Inês Queiroz-Garcia, ${ }^{1}$ \\ Filipa Pimenta, ${ }^{1}$ Rui Miguel Costa, ${ }^{1}$ Melissa A Day, ${ }^{2,3}$ José Pais-Ribeiro, ${ }^{1}$ \\ Mark P Jensen ${ }^{2}$
}

To cite: Ferreira-Valente A, Jarego M, Queiroz-Garcia I, et al. Prayer as a pain intervention: protocol of a systematic review of randomised controlled trials. BMJ Open 2021;11:e047580. doi:10.1136/ bmjopen-2020-047580

- Prepublication history and additional supplemental material for this paper are available online. To view these files, please visit the journal online. To view these files, please visit the journal online (http://dx.doi org/10.1136/bmjopen-2020047580).

Received 04 December 2020 Accepted 17 June 2021

Check for updates

(c) Author(s) (or their employer(s)) 2021. Re-use permitted under CC BY-NC. No commercial re-use. See rights and permissions. Published by BMJ.

${ }^{1}$ William James Center for Research, ISPA - University Institute, Lisbon, Portugal ${ }^{2}$ Department of Rehabilitation Medicine, University of Washington, Seattle, Washington, USA

${ }^{3}$ School of Psychology, The University of Queensland, Brisbane, Queensland, Australia

Correspondence to Dr Alexandra Ferreira-Valente; mafvalente@gmail.com

\section{ABSTRACT}

Background Pain is a universal experience and the most common reason for seeking healthcare. Inadequate pain management negatively impacts numerous aspects of patient health. Multidisciplinary treatment programmes, including psychosocial interventions, are more useful for pain management than purely biomedical treatment alone. Recently, researchers showed increasing interest in understanding the role of spirituality/religiosity and spiritual/religious practices on pain experience, with engagement in religious practices, such as prayer, showing to positively impact pain experience in religious individuals. This systematic review will seek to summarise and integrate the existing findings from randomised controlled trials assessing the effects of prayer and prayerbased interventions on pain experience.

Methods The systematic review procedures and its report will follow the Preferred Reporting Items for Systematic reviews and Meta-Analyses statement. Electronic searches in nine databases (Web of Science Core Collection, MEDLINE, SCIELO Citation Index, PubMed, Cochrane Central Register of Controlled Clinical Trial, PsycINFO, Scopus, LILACS and Open-SIGLE) will be performed to identify randomised controlled trials of prayer-based interventions. Two independent researchers will assess studies for inclusion and extract data from each paper. Risk of bias assessment will be assessed independently by two reviewers based on the Consolidated Standards of Reporting Trials statement. Qualitative synthesis of the body of research will be conducted using a narrative summary synthesis method. Meta-analysis will be limited to studies reporting on the same primary outcome. Formal searches are planned to start in June 2021. The final report is anticipated to be completed by September 2021. Discussion Findings will be useful to (1) understand the condition of our knowledge in this field and (2) provide evidence for prayer effectiveness in reducing pain intensity and pain-related stress and increasing pain tolerance in adults experiencing acute or chronic pain.

PROSPERO registration number CRD42020221733.

\section{INTRODUCTION}

Pain-defined by the International Association for the Study of Pain as an 'unpleasant sensory and emotional experience associated with, or resembling that associated

\section{Strengths and limitations of this study}

- This is a comprehensive systematic review focusing the effects of participatory prayer on pain-related outcomes (despite the type of pain), including studies published in peer-reviewed indexed journals and on the grey literature regardless of the date of publication.

- Preferred Reporting Items for Systematic reviews and Meta-Analyses (PRISMA) statement guidelines will be followed to conduct this review, and PRISMA Protocol checklist was followed to report the protocol of this systematic review, to ensure quality in all aspects of study planning, execution and reporting.

- Risk of bias assessment, assessment of reporting and publication bias, and evaluation of the confidence in cumulative evidence will be performed, respectively, based on the Consolidated Standards of Reporting Trials statement, the Egger's regression test method, and on the Grading of Recommendations Assessment, Development and Evaluation approach.

- Main strengths of the study are its comprehensiveness, including studies and the grey literature published in five languages despite the date of publication, and the use of well-established and standardised methods to perform qualitative synthesis, meta-analysis, risk of bias assessment and metabias assessment.

- We may not be able to conduct the anticipated meta-analysis due to methodological heterogeneity between the included studies and between the primary outcomes' measures used in the different studies.

with, actual or potential tissue damage ${ }^{1}$-is a universal experience. ${ }^{2}$ Despite its unpleasantness, pain-except when associated with many chronic pain conditions-usually has an adaptive purpose. It is a sign that physical damage might be occurring that threatens the organism's well-being. ${ }^{3}$ Pain is the most common reason for seeking healthcare, ${ }^{4}$ and inadequate management of acute pain negatively impacts numerous aspects of patient 
health. This includes reduced quality of life, impaired sleep, impaired physical function and increased risk of developing chronic pain. ${ }^{5}$ In short, pain remains a significant health issue, and its management a priority worldwide..$^{5-8}$

Because pain is a multidimensional and subjective experience, researchers and clinicians have worked to clarify the role that biological (eg, hormone levels, extent of physical damage), psychological (eg, beliefs, coping responses) and social factors (eg, social support) have in influencing the experience and impact of pain. ${ }^{9}$ The findings from this research have informed the development of pain management multidisciplinary treatment programmes, including psychosocial interventions, which are more effective in managing chronic pain than treatments that focus only on biomedical factors. ${ }^{9-11}$

More recently, researchers have become interested in understanding the association between pain, adjustment to pain, spirituality (and spiritual practices) and religiosity (and religious practices). Taken together, findings regarding the benefits of spiritual/religiosity in people with pain are encouraging. A recent systematic review summarised the evidence relative to the extent to which measures of spiritual/religiosity are associated with painrelated outcomes, pain-related beliefs and pain-coping responses in people with chronic pain. ${ }^{12}$ The results from this review suggested that, regardless of the great variety of measures used to assess spirituality/religiosity and pain-related outcomes which limit the drawing of strong conclusions, a number of religiosity/spirituality domains are associated with lower pain (intensity and interference) and with better psychological function in people with chronic pain.

The findings from the body of research on the effects of specific spiritual and religious practices suggest that at least some of these practices may be useful resources in the context of pain care. For example, preliminary findings indicate that people who are more engaged with meaningful spiritual and religious practices-such as spiritual meditation and prayer-can tolerate pain better; ${ }^{1314}$ that is, those more engaged in these practices evidence a greater willingness to accept pain than those less engaged in these practices. ${ }^{15}$ Prayer-defined as a form of spiritual communication between a human being with a deity (eg, God) or with an object of prayer ${ }^{16}$ - is among the spiritual and religious practices that have the potential to explain the beneficial effects of spirituality/religiosity on pain.

Prayer is among the most common and valued practices of individuals of both Western and Eastern countries of different religious denominations, even if they are not actively involved members of a religious tradition. ${ }^{14}$ Prayer is a complex and multidimensional activity. Although there is a general consensus about its definition, there is not a dominant theoretical model or consistent approach to classify or measure different types of prayer. ${ }^{1718}$ In fact, previous research on the effects of prayer-based intervention for pain often fail to provide a clear definition of what is meant by 'prayer'. These studies show a high degree of theoretical and methodological heterogeneity relative to the taxonomy of prayer employed, and its measurement. ${ }^{17-19}$ Although this heterogeneity makes comparisons across studies difficult, it also may account, at least in part, for the inconsistent findings regarding the effects of prayer-based interventions on health-related and painrelated outcomes, given the possibility that different types of prayer may have different effects on these outcomes.

Prayer may be private (or individual, ie, an individual prays alone) or communal (ie, an individual prays with another person or group of people), with respect to the number of people engaged in the prayer. ${ }^{1620}$ With respect to content, prayer can be of petition (or supplication, ie, a request to the deity's or object of prayer's intervention towards oneself or towards another person or group of people), thanksgiving (ie, expressing gratefulness), adoration (ie, worshiping or praising the deity or object of prayer), confession (ie, recognition of sins, misdeeds or faults), reception (ie, waiting passively for the deity's or object of prayer's inspiration, wisdom and understanding), meditation (ie, reflecting about a sacred text or a religious topic) or ritualistic (ie, reading or reciting by memory a prayer or religious text). ${ }^{172021}$ Prayer can also be participatory or distant intercessory, with respect to the targeted beneficiary of the prayer. For participatory prayer, the targeted beneficiary of the prayer is one or more of the people doing the praying. For distant prayer, however, the targeted beneficiary of the praying is someone other than the person or people doing the praying. Of note, it is not the number of individuals involved in the praying (ie, just one individual or more than one individual) that determines the content or beneficiary of the prayer. Participatory prayer, for example, may be private (or individual) or communal (ie, dyadic or group-prayer) where the beneficiary of the prayer is/are either the individual or the people praying together. Distant petition prayer, either private or communal, where the beneficiary of the prayer is/are someone other than the person or people doing the praying, is often called distant intercessory prayer.

Although previous research suggests the effects of distant intercessory prayer on health-related and painrelated outcomes are weak, ${ }^{18} 22{ }^{23}$ previous findings relative to participatory prayer (ie, individual, dyadic or group-based prayer where the targeted beneficiary of the prayer is one or more of the people doing the praying) generally support stronger beneficial effects, and support its potential usefulness for patient care in the context of pain. For example, one study ${ }^{24}$ with Muslim women who underwent a caesarean section showed that, compared with a no treatment control, women randomised to an Islamic prayer condition reported significantly lower pain intensity scores (ie, the magnitude of the experienced pain $^{25}$ ) at 3 hours and 6 hours after the intervention. ${ }^{24}$ Another study with patients who underwent gastrointestinal elective surgery, suggested that prayer as an adjunctive intervention to the use of an analgesic medication contributed to reduced postoperatory pain intensity to a greater extent than the use of an analgesic medication 
alone. ${ }^{26}$ Similar results were also found by Rahman and colleagues $^{27}$ when comparing the postoperatory pain intensity decrease of patients submitted to an emergency appendectomy in the prayer condition, to that observed in patients in the music-therapy and in the control conditions. Although these and other studies suggest that spiritual/religiosity and spiritual/religious practices contribute to reduce self-reported pain-related distress in people with pain, ${ }^{12}$ the results of studies focusing the effects of prayer-based interventions on objective stress responses are inconsistent; that is, a beneficial effect of prayer on self-reported pain-related stress and on objective stress responses (eg, respiration and heart rate) is not always found. ${ }^{24} 28$

A recent systematic review summarised the evidence relative to the benefits of active personal participatory prayer as an intervention implemented on-site for pain. ${ }^{19}$ Results from this review suggested that prayer appears to be useful in reducing pain intensity and increasing pain tolerance, especially among religious people undergoing surgery or a painful procedure and engaging in an active prayer to a deity. ${ }^{19}$ However, this systematic review was limited to studies published in English between the years 2000 and 2019, in peer-reviewed journals indexed in one or more of only four databases (PubMed, Web of Science, PsychINFO and Scopus). Thus, this review might have missed an important number of studies that (a) were published in languages other than English, (b) were published before the year 2000, and (c) were published in journals indexed in other relevant databases or present in the grey literature. This systematic review was also restricted to the effects of private (individual) prayer on pain, thus excluding studies evaluating the effects of communal (either dyadic or group) prayer, which is a practice of many religions. Furthermore, this previous review (a) did not provide a qualitative review of the findings based on a standardised and well-established method for qualitative synthesis of the findings and (b) did not conduct a quality assessment of the studies included in the review. A comprehensive systematic review focusing the effects of both private (individual) and communal (dyadic and group) prayer on acute and chronic painrelated outcomes, including studies published both in peer-reviewed indexed journals and in the grey literature regardless of the date of publication, using a standardised method for performing qualitative data analysis and metanalysis, and reporting on the characteristics and assessing the methodological quality of the included studies, would be useful for a more comprehensive and complete understanding of the usefulness of prayer as a pain management intervention. Hence, this study aims to perform such a systematic review.

\section{Study aims}

The planned systematic review seeks to summarise and integrate the existing findings from randomised controlled trials assessing the effects of participatory prayer-based interventions (including private and communal-based participatory prayer) on pain-related outcomes (ie, pain intensity, pain tolerance and painrelated stress). Specifically, this systematic review seeks to examine the extent to which prayer affects pain-related outcomes in adults with acute or chronic pain while including studies published in English, German, French, Spanish, Italian or Portuguese (ie, the languages understood by one or more authors on the team), searched in nine databases (including one grey literature database), performing qualitative data analysis and including an assessment of the studies' methodological quality using standardized and well-established methods, and making recommendations for future research in this area. Given the subjective nature of pain's experience, this systematic review will be limited to studies that include a self-report of pain intensity, pain tolerance, and/or pain-related stress as a primary outcomes.

\section{METHOD \\ Review protocol}

The present systematic review will be conducted in accordance with the Preferred Reporting Items for Systematic reviews and Meta-Analyses (PRISMA) statement for systematic reviews and meta-analysis protocols. ${ }^{29}$ Likewise, the PRISMA Protocol checklist will be used to guide the protocol of this systematic review. ${ }^{30}$

\section{Review participants}

Participants will be adult individuals experiencing pain, regardless of the cause of pain, pain duration or pain location.

\section{Eligibility criteria}

Articles will be included in this systematic review if they (1) studied participants who are adults (ie, $\geq 18$ years old); (2) studied participants with pain, regardless of its duration or aetiology; (3) included at least one quantitative measure of self-reported pain intensity, pain tolerance and/or pain-related stress; (4) assessed the effects of private (individual) or communal (dyadic or group) participatory prayer-based interventions; (5) compared the intervention group to a control group (eg, alternative treatment(s), treatment as usual or both); (6) used a randomised controlled trial design; and (7) were published in English, German, French, Spanish, Italian or Portuguese. Although including only studies written in these languages might be viewed as restrictive, the authors are only proficient in these languages and do not have the resources to include articles written in other languages. At least two searches will be conducted; one for the first draft of the review paper and a second search to update the review paper just prior to submission. Papers reporting the results of qualitative, cohort, case-control and cross-sectional studies and papers focusing on spiritual practices, complementary and integrative health interventions other than prayer (eg, meditation, Reiki 
and yoga) or distant intercessory prayer will be excluded from the systematic review.

\section{Patient and public involvement}

No patient was involved in either the design or planning phase of this study.

\section{Search strategy}

The systematic review protocol specifies and documents the search strategy, methods and inclusion/exclusion criteria of the articles in advance. The search strategies that will be used to identify relevant publications will be customised to suit each database. The search terms will be (1) Religion OR Prayer; AND (2) Pain; AND (3) Pain intensity OR Pain tolerance OR Stress. The search terms will be used in various combinations, considering synonyms and MESH entries (see online supplemental file 1).

We will search nine databases for the relevant publications: Web of Science Core Collection (Clarivate Analytics), MEDLINE (via Web of Science, Clarivate Analytics), SCIELO Citation Index (via Web of Science, Clarivate Analytics), PubMed, Cochrane Central Register of Controlled Clinical Trial (via OvidSP), PsycINFO, Scopus, LILACS and Open-SIGLE. The unpublished literature will also be searched in clinical trial registry platforms, such as the ClinicalTrials.gov and the International Standard Randomised Controlled Trial Number (ISRCTN) registry. Then, to identify additional articles not identified during the database search, references list of articles included in the review and review articles found by the search will be hand searched. Preliminary searches were planned to start in December 2020. Formal searches are planned to be completed in July 2021.

\section{Co-primary outcomes}

Studies will be included in the systematic review if they include at least one quantitative measure of pain intensity, pain tolerance or pain-related stress. The following are the commonly used measures assessing these variables.

Among the most common measures of pain intensity used by researchers and clinicians are the Visual Analogue Scale $\left(\mathrm{VAS}^{31}\right)$ and related versions of this scale (eg, mechanical VAS and digital VAS). Other commonly used measures of pain intensity ${ }^{32}$ are the $0-10$ Numerical Rating Scale (NRS), the Verbal Rating Scale (VRS) and the Faces Pain Scale-Revised ${ }^{33}$. Each of these measures is adequately valid and reliable as a measure of pain intensity, and patients in Western countries tend to prefer VRS and NRS over VAS. ${ }^{32}$ Pain tolerance is commonly operationalised as the length (in seconds) that an individual is willing to experience an aversive stimulation. ${ }^{34-36}$

Finally, perceived stress is usually assessed with the: (a) Perceived Stress Scale ${ }^{37}$, a 10-item scale that asks about people's feelings and thoughts regarding the passing month, on a scale from 0 (never) to 4 (very often); (b) stress scale of Depression, Anxiety and Stress Scale $\left(\mathrm{DASS}^{38}\right)$, a 14-item scale where subjects are asked to use 4-point severity/frequency scales to rate the extent to which they have experienced each state over the past week and (c) 21-item version (DASS-21), which consist of a 7-item scale similar to the previous. In contrast, objective stress is usually measured through salivary cortisol, heart rate variability or blood pressure measurement and analysis. ${ }^{39-41}$

\section{Study selection, data management and data extraction}

First, all references identified during the database search will be inserted into Zotero Software. ${ }^{42}$ Second, crossreferences and duplicates will be deleted. Then, two independent reviewers (MJa and IQ-G) will screen the titles, abstracts and keywords of the identified studies for eligibility based on the eligibility criteria listed above. Full texts of the manuscripts meeting the eligibility criteria and those with an uncertainty of inclusion based on the abstracts will be independently read by the same reviewers to confirm inclusion or exclusion. Discrepancies will be settled during a consensus meeting. In cases in which consensus is not achieved, a third reviewer will be consulted (AF-V). The studies' inclusion or exclusion will be recorded on a self-designed data extraction form in Microsoft Excel 10.

Third, detailed data from the studies meeting the inclusion criteria will be extracted on a Microsoft Excel extraction sheet by two authors (MJa and IQ-G). Data to be extracted include: (1) authors and year of publication; (2) country of study; (3) study participant (type of painchronic or acute-and pain aetiology); (4) sample size; (5) study participants' age (mean, SD); (6) percentage of female participants; (7) site of pain; (8) study participants' religious denomination and self-reported spirituality (eg, self-proclaimed as 'spiritual'); (9) study participants' type of religious practice (religious and a practitioner, religious but not a practitioner); (10) study participants' attitudes toward the religion (negative attitude towards deity vs positive attitude towards deity); (11) length and intensity (frequency and length of sessions) of prayer-based intervention; (12) type of prayer [private or communal (ie, dyadic or group) participatory prayer]; (13) content of prayer (petition, thanksgiving, adoration, confession, reception, meditative or ritualistic); (14) level of engagement and emotional involvement with the prayer-based intervention; (15) type and characteristics of the comparator or control condition; (16) self-reported pain intensity; (17) pain tolerance and (18) pain-related stress. Data (mean, SD or effect sizes) on the primary outcomes will be extracted. Whenever possible, the rates of meaningful reductions in pain intensity-that is, from responder analyses-will also be extracted; more specifically, the rates by which different groups obtained a $30 \%$ and a 50\% reduction in pain intensity. Any discrepancies in the data collected will be resolved by discussion and consensus. In the event that consensus is not achieved, a third author (AF-V) will be consulted. Full-text screening and data extraction are planned to be completed in July 2021. 


\section{Risk of bias assessment}

The risk of bias of the studies included in the present systematic review will be evaluated based on the Consolidated Standards of Reporting Trials statement. Risk of bias will be assessed by two independent reviewers (MJa and IQ-G) and discrepancies will be settled during a consensus meeting. If a consensus is not reached, a third reviewer (AF-V) will be consulted. Risk of bias assessment is anticipated to be completed in August 2021.

\section{Data analysis plan}

Qualitative synthesis. A qualitative synthesis of the body of research will be conducted, ${ }^{43}$ describing the methodological characteristics of the studies included in this systematic review, their strengths and limitations, and each study's results regarding the effects of private or communal participatory prayer in reducing pain intensity and pain-related stress and in increasing pain tolerance in adults experiencing pain, compared with treatment as usual or no treatment. Outcomes from private participatory prayer and from communal participatory prayer will be summarised and examined separately. To summarise the evidence, we will implement a narrative summary synthesis method based on the framework developed by the UK Economic and Social Research Council to the conduct of narrative synthesis in systematic reviews, ${ }^{44}$ modified to fit our purposes. Following the referred guidance, the synthesis process will involve three elements: (1) developing a preliminary synthesis of findings of included studies; (2) exploring relationships in the data; and (3) assessing the robustness of the synthesis.

Meta-analysis. Outcomes from private participatory prayer and from communal participatory prayer will be analysed separately. If at least two included articles present data on a single primary outcome, then metaanalysis will be performed. ${ }^{45}$ End-point scores will be computed as expressed mean differences or standardised mean difference for each study for primary outcomes, with associated 95\% CIs. Data extracted in the articles will be transformed and presented in Cohen's $d$ effect size with $95 \%$ CI when possible using the Campbell Collaboration online calculator. ${ }^{46}$ The Cohen's $d$ effect size thresholds we will use small (0.20), medium $(0.50)$, large $(0.80)$ and very large $(1.30){ }^{47}$ Data analysis is anticipated to be completed in August 2021.

\section{Assessment of reporting biases}

We will use funnel plots to evaluate the potential reporting bias and small-study effects when there are $>10$ studies in the meta-analysis. Quantification of publication bias will be done using Egger's regression test method. ${ }^{48}$ Assessment of reporting biases is anticipated to be completed in August 2021.

\section{Confidence in cumulative evidence}

Included studies will be critically appraised and synthesised in terms of methodological quality along with the relevance, strength and limitations of the evidence presented. The similarities and differences between the studies (study characteristics, design and execution) will be described and their potential impact on study outcome will be discussed. The strength of the body of evidence will be assessed using the Grading of Recommendations Assessment, Development and Evaluation approach.

\section{DISCUSSION}

This systematic review will provide evidence for prayer effectiveness in reducing pain intensity and pain-related stress and increasing pain tolerance in adults experiencing acute or chronic pain. This allows for a more considerate and insightful choice when advising pain patients with effective coping strategies.

\section{ETHICS AND DISSEMINATION}

Ethics approval is not required for this review because it relies on secondary data. Any modification to the systematic review protocol will be submitted to review and approval of the PROSPERO registry and described in the final report of the systematic review. The systematic review findings will be published in a peer-reviewed indexed journal and presented at a pain international scientific meeting. It is anticipated that the final report will be completed by September 2021.

\section{Twitter Alexandra Ferreira-Valente @Alexand79646363}

Contributors AF-V conceived the study idea, and together with MJa, MJe and MD developed the design of the protocol. IQ-G, FP, RC, JP-R and MJe substantially contributed to the review and refinement of the review design and protocol. AF-V and IQ-G planned the data analysis. AF-V and MJa wrote the first draft of the manuscript. All authors critically reviewed the manuscript and approved the final version. AF-V, MJa and IQ-G will conduct the review procedures. AF-V is the principal investigator, obtained funding and is the guarantor of the review.

Funding This study was supported by a Foundation BIAL Grant for Scientific Research (grant number: 188/18). AF-V has received a grant from the Portuguese Foundation for Science and Technology (grant number: SFRH/BPD/121452/2016).

Competing interests None declared.

Patient consent for publication Not required.

Provenance and peer review Not commissioned; externally peer reviewed.

Supplemental material This content has been supplied by the author(s). It has not been vetted by BMJ Publishing Group Limited (BMJ) and may not have been peer-reviewed. Any opinions or recommendations discussed are solely those of the author(s) and are not endorsed by BMJ. BMJ disclaims all liability and responsibility arising from any reliance placed on the content. Where the content includes any translated material, BMJ does not warrant the accuracy and reliability of the translations (including but not limited to local regulations, clinical guidelines, terminology, drug names and drug dosages), and is not responsible for any error and/or omissions arising from translation and adaptation or otherwise.

Open access This is an open access article distributed in accordance with the Creative Commons Attribution Non Commercial (CC BY-NC 4.0) license, which permits others to distribute, remix, adapt, build upon this work non-commercially, and license their derivative works on different terms, provided the original work is properly cited, appropriate credit is given, any changes made indicated, and the use is non-commercial. See: http://creativecommons.org/licenses/by-nc/4.0/. 
ORCID iD

Alexandra Ferreira-Valente http://orcid.org/0000-0002-3684-5561

\section{REFERENCES}

1 Raja SN, Carr DB, Cohen M, et al. The revised international association for the study of pain definition of pain: concepts, challenges, and compromises. Pain 2020;161:1976-82.

2 Wierzbicka A. Is pain a human universal? A cross-linguistic and cross-cultural perspective on pain. Emotion Review 2012;4:307-17.

3 Schneiderman N, Ironson G, Siegel SD. Stress and health: psychological, behavioral, and biological determinants. Annu Rev Clin Psychol 2005;1:607-28.

4 National Center for Complementary and Integrative Health. Complementary, Alternative, or Integrative Health: What's In a Name? Complement Altern. Integr. Health What's Name, 2020. Available: https://nccih.nih.gov/health/integrative-health

5 Sinatra R. Causes and consequences of inadequate management of acute pain. Pain Med 2010;11:1859-71

6 Dunwoody CJ, Krenzischek DA, Pasero C, et al. Assessment, physiological monitoring, and consequences of inadequately treated acute pain. Pain Management Nursing 2008:9:11-21.

7 Breivik H, Collett B, Ventafridda V, et al. Survey of chronic pain in Europe: prevalence, impact on daily life, and treatment. Eur J Pain 2006;10:287.

8 Morlion B, Walch H, Yihune G, et al. The pain associates' international network initiative: a novel practical approach to the challenge of chronic pain management in Europe. Pain Pract 2008;8:473-80.

9 Gatchel RJ, Peng YB, Peters ML, et al. The biopsychosocial approach to chronic pain: scientific advances and future directions. Psychol Bull 2007;133:581-624.

10 Koenig HG, Weiner DK, Peterson BL, et al. Religious coping in the nursing home: a biopsychosocial model. Int J Psychiatry Med 1997;27:365-76.

11 Gatchel RJ. Comorbidity of chronic pain and mental health disorders: the biopsychosocial perspective. Am Psychol 2004;59:795-805.

12 Ferreira-Valente A, Sharma S, Torres S. Does Religiosity/Spirituality play a role in function, pain-related beliefs, and coping in patients with chronic pain? A systematic review. J Relig Health. [Epub ahead of print: 18 Sep 2019].

13 Lysne CJ, Wachholtz AB. Pain, spirituality, and meaning making: what can we learn from the literature? Religions 2010;2:1-16.

14 Dezutter J, Wachholtz A, Corveleyn J. Prayer and pain: the mediating role of positive re-appraisal. J Behav Med 2011;34:542-9.

15 Merskey H, Bogduk N. Classification of chronic pain: descriptions of chronic pain syndromes and definitions of pain terms. 2nd edn Seattle: IASP Press, 1994.

16 Anderson JW, Nunnelley PA. Private Prayer associations with depression, anxiety and other health conditions: an analytical review of clinical studies. Postgrad Med 2016;128:635-41.

17 Pérez JE, Rex Smith A, Norris RL, et al. Types of Prayer and depressive symptoms among cancer patients: the mediating role of rumination and social support. J Behav Med 2011;34:519-30.

18 Masters KS, Spielmans GI. Prayer and health: review, meta-analysis, and research agenda. J Behav Med 2007;30:329-38.

19 Illueca M, Doolittle BR. The use of Prayer in the management of pain: a systematic review. J Relig Health 2020;59:681-99.

20 Baesler EJ. A model of interpersonal Christian Prayer, 1999.

21 Laird SP, Snyder CR, Rapoff MA, et al. Measuring private prayer: Development, validation, and clinical application of the Multidimensional Prayer Inventory. Int J Psychol Relig 2004;14:251-72

22 Masters KS, Spielmans GI, Goodson JT. Are there demonstrable effects of distant intercessory Prayer? A meta-analytic review. Ann Behav Med 2006;32:21-6.

23 Vincent S. Livin' on a prayer: An analysis of intercessory prayer studies 2016;3:9.

24 Beiranvand S, Noparast M, Eslamizade N, et al. The effects of religion and spirituality on postoperative pain, hemodynamic functioning and anxiety after cesarean section. Acta Med Iran 2014;52:909-15.
25 Jensen MP, Karoly P. Measurement of cancer pain via patient selfreport. In: Current and emerging issues in cancer pain: research and practice. New York: Raven Press, 1993: 193-218.

26 Dehkordi A, Fatehi D, Solati K. Analgesic plus Prayer versus analgesic alone. Effect of Prayer on intensity of postoperative pain, anxiety and physiological indices in surgical patients. A randomized clinical trial. Heroin Addict Relat Clin Probl 2016;18:13-20.

27 Rahman FS, Yahya N, Md Din NM, et al. The comparative effects of listening to Prayer Recitation and music therapy intraoperatively on postoperative pain. Imjm 2020;17.

28 Sollgruber A, Bornemann-Cimenti H, Szilagyi I-S, et al. Spirituality in pain medicine: a randomized experiment of pain perception, heart rate and religious spiritual well-being by using a single session meditation methodology. PLoS One 2018;13:e0203336.

29 Liberati A, Altman DG, Tetzlaff J, et al. The PRISMA statement for reporting systematic reviews and meta-analyses of studies that evaluate health care interventions: explanation and elaboration. PLoS Med 2009;6:e1000100.

30 Moher D, Shamseer L, Clarke M, et al. Preferred reporting items for systematic review and meta-analysis protocols (PRISMA-P) 2015 statement. Syst Rev 2015;4:1.

31 Huskisson E. Visual analogue scales. In: Pain measurement and assessment. New York: Raven Press, 1983: 33-7.

32 Jensen MP. Measurement of pain. In: Bonica's management of pain. Philadelphia: Wolters Kluwer, 2019: 974-1037.

33 Hicks CL, von Baeyer CL, Spaffor PA, et al. The faces pain ScaleRevised: toward a common metric in pediatric pain measurement. Pain 2001;93:173-83.

34 Feuille M, Pargament K. Pain, mindfulness, and spirituality: a randomized controlled trial comparing effects of mindfulness and relaxation on pain-related outcomes in migraineurs. $J$ Health Psychol 2015;20:1090-106.

35 Gonçalves JPdeB, Lucchetti G, Menezes PR, et al. Complementary religious and spiritual interventions in physical health and quality of life: a systematic review of randomized controlled clinical trials. PLoS One 2017;12:e0186539.

36 Samulowitz A, Gremyr I, Eriksson E, et al. "Brave Men" and "Emotional Women": A Theory-Guided Literature Review on Gender Bias in Health Care and Gendered Norms towards Patients with Chronic Pain. Pain Research and Management 2018;2018:1-14.

37 Cohen S, Tyrrell DAJ, Smith AP. Negative affect, and susceptibility to the common cold. J Pers Soc Psychol 1993;64:131-40.

38 Lovibond SH, Lovibond PF. Manual for the depression anxiety stress scales. 2nd ed. Sydney: Psychology Foundation of Australia, 1995.

39 Laborde S, Mosley E, Thayer JF. Heart rate variability and cardiac vagal tone in psychophysiological research - recommendations for experiment planning, data analysis, and data reporting. Front Psychol 2017;08.

40 Schwabe L, Haddad L, Schachinger H. HPA axis activation by a socially evaluated cold-pressor test. Psychoneuroendocrinology 2008;33:890-5

41 Schwartz L, Slater MA, Birchler GR. Interpersonal stress and pain behaviors in patients with chronic pain. J Consult Clin Psychol 1994;62:861-4.

42 Zotero Software. U.S. Roy Rosenzweig center for history and new media. Zotero Software. U.S, 2016.

43 Eden J, Levit L, Berg A. Finding what works in health care: Standards for systematic reviews. Washington, DC: National Academies Press, 2011.

44 Popay J, Roberts H, Sowden A. Guidance on the conduct of narrative synthesis in systematic reviews. ESRC Methods Programme, 2006.

45 Davey J, Turner RM, Clarke MJ, et al. Characteristics of metaanalyses and their component studies in the Cochrane database of systematic reviews: a cross-sectional, descriptive analysis. BMC Med Res Methodol 2011;11:160.

46 Lipsey MW, Wilson DB. Practical meta-analysis effect size calculator Campbell Collab, 2001. Available: https://www.campbellcollabor ation.org/escalc/html/EffectSizeCalculator-Home.php

47 Cohen J. Statistical power analysis for the behavioral sciences. 2nd ed. Hillsdale: Lawrence Erlbaum, 1988.

48 Lin L, Chu H. Quantifying publication bias in meta-analysis: quantifying publication bias. Biometrics 2018;74:785-94. 